\title{
Bounds on large extra dimensions from the simulation of black hole events at the LHC
}

\author{
Shaoqi Hou, ${ }^{a}$ Benjamin Harms $^{a}$ and Marco Cavaglià ${ }^{b}$ \\ ${ }^{a}$ Department of Physics $\&$ Astronomy, The University of Alabama, \\ Tuscaloosa, Alabama 35487/0324, U.S.A. \\ ${ }^{b}$ Department of Physics $\&$ Astronomy, The University of Mississippi, \\ University, Mississsippi 38677/1848, U.S.A. \\ E-mail: shou@crimson.ua.edu, bharms@ua.edu, cavaglia@phy.olemiss.edu
}

\begin{abstract}
If large extra dimensions exist, the Planck scale may be as low as a $\mathrm{TeV}$ and microscopic black holes may be produced in high-energy particle collisions at this energy scale. We simulate microscopic black hole formation at the Large Hadron Collider and compare the simulation results with recent experimental data by the Compact Muon Solenoid collaboration. The absence of observed black hole events in the experimental data allows us to set lower bounds on the Planck scale and various parameters related to microscopic black hole formation for a number $(3-6)$ of extra dimensions. Our analysis sets lower bounds on the fundamental Planck scale ranging from $0.6 \mathrm{TeV}$ to $4.8 \mathrm{TeV}$ for black holes fully decaying into Standard Model particles and $0.3 \mathrm{TeV}$ to $2.8 \mathrm{TeV}$ for black holes settling down to a remnant, depending on the minimum allowed black hole mass at formation. Formation of black holes with mass less than $5.2 \mathrm{TeV}$ to $6.5 \mathrm{TeV}$ (SM decay) and $2.2 \mathrm{TeV}$ to $3.4 \mathrm{TeV}$ (remnant) is excluded at 95\% C.L. Our analysis shows consistency with and difference from the CMS results.
\end{abstract}

KEywords: Phenomenology of Large extra dimensions, Monte Carlo Simulations

ARXIV EPRINT: 1507.01632 


\section{Contents}

1 Introduction 1

2 Black hole formation in particle collisions 3

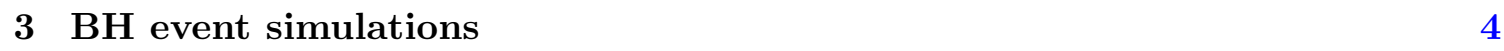

4 Results 5

5 Conclusions $\quad 9$

$\begin{array}{ll}\text { A Procedure for determining } M_{*} \text { and } M_{\min } \text { limits } & 10\end{array}$

\section{Introduction}

The Standard Model of Elementary Particles (SM) is one of the most successful hypotheses in physics [1]. However, the SM fails to explain the hierarchy problem, i.e., the huge gap between the electroweak scale $\left(M_{\mathrm{EW}} \sim 1 \mathrm{TeV}\right)$ and the observed Planck scale $\left(M_{\mathrm{Pl}} \sim 10^{16} \mathrm{TeV}\right)$. The ADD model [2-4] provides a way to solve the hierarchy problem by introducing a number $n$ of large, compactified spatial dimensions (LEDs). Gravitons can propagate in the $D(=n+4)$-dimensional space-time bulk. SM particles are confined to the 4-dimensional brane. Assuming compactification on a torus with equal radii $R$, the observed Planck mass $M_{\mathrm{Pl}}$ is related to the 4-dimensional fundamental Planck mass $M_{*}$ by $M_{\mathrm{Pl}}^{2}=(2 \pi R)^{n} M_{*}^{D-2}$. If $R$ is sufficiently large, the fundamental Planck mass $M_{*}$ may be as low as a few TeVs.

If the ADD model is realized in nature, strong gravitational effects should manifest themselves in physical processes at the $\mathrm{TeV}$ scale. Gravitational phenomena at the $\mathrm{TeV}$ scale could include, for example, graviton and Kaluza-Klein (KK) mode production in particle scattering [5, 6] and even microscopic black hole (BH) formation [7, 8]. The Large Hadron Collider (LHC), operating at a center of mass energy of several TeVs, can be used to probe the appearance of these new physical processes and shed light on the existence of large extra dimensions [1, 9-12].

To date, experimental results have not confirmed the existence of large extra dimensions (see, eg. ref.s $[13,15-17,37,46,47]$ ). These null results set upper bounds on the size of the large extra dimensions, or equivalently, lower bounds on the the fundamental Planck scale. The ADD model with one LED requires the size of the LED to be of the order of $10^{9} \mathrm{~km}$ and is macroscopically ruled out because no deviations from Newtonian gravity have been observed at the solar system scale. Observations of neutron stars by the Fermi Large Area Telescope [15] rule out ADD models with two LEDs. Constraints on 


\begin{tabular}{|cccccccccccc|}
\hline \multicolumn{110}{|c|}{ References } \\
\cline { 2 - 12 }$y$ & {$[25]$} & {$[26]$} & {$[27,28]^{\dagger}$} & {$[29]^{\dagger}$} & {$[30]^{\dagger}$} & {$[31]$} & {$[32]$} & {$[33]^{\dagger}$} & {$[34]^{\dagger}$} & {$[35]$} & {$[36]$} \\
\hline 3 & 3.16 & 4.29 & 1.20 & 2.05 & 1.20 & 4.77 & 4.11 & 1.16 & 0.81 & 2.30 & 2.12 \\
4 & 2.84 & 3.71 & 1.17 & 2.00 & 1.17 & 3.97 & 3.56 & 1.13 & 0.79 & 2.20 & 2.13 \\
5 & 2.65 & 3.31 & 1.12 & 1.92 & 1.12 & 3.73 & 3.24 & 1.08 & 0.76 & 2.04 & 2.14 \\
6 & 2.58 & 3.12 & 1.07 & 1.84 & 1.07 & 3.53 & 2.96 & 1.03 & 0.72 & 2.00 & 2.17 \\
\hline
\end{tabular}

Table 1. The observed lower limits on $M_{D}$ from collider experiments in TeV.

space-times with three large extra dimensions from astrophysical and cosmological experiments are generally very stringent, although they typically suffer from large systematic errors. The observation of Supernova SN1987A sets a lower limit on $M_{D}$ of $2.4 \mathrm{TeV}$ for $n=3$ [16], where the reduced Planck mass $M_{D}$ is related to $M_{*}$ by [9],

$$
M_{D}=\left[\frac{(2 \pi)^{n}}{8 \pi}\right]^{\frac{1}{n+2}} M_{*} .
$$

Neutron star-derived limits constrain $M_{D}$ to be larger than $76 \mathrm{TeV}$ for $n=3$ [17]. Nonobservation of perturbative processes predicted by LED models in collider experiments [25][36] provide less stringent, albeit more accurate limits on $M_{D}$ or the string scale $\Lambda_{\mathrm{T}}$, which is related to $M_{D}$ by [18]

$$
\Lambda_{\mathrm{T}}=2 \sqrt{\pi}\left[\Gamma\left(\frac{n}{2}\right)\right]^{1 /(n+2)} M_{D}
$$

Current limits on $M_{D}$ (in units of $\mathrm{TeV}$ ) from these experiments are shown in table 1, where references labeled by $\dagger$ indicate lower bounds on $M_{D}$ derived from constraints on the string scale $\Lambda_{\mathrm{T}}$, and the remaining ones indicate lower bounds on $M_{D}$ directly. The search for the extinction of QCD jet production by the Compact Muon Solenoid (CMS) Collaboration provides an additional lower limit of $3.3 \mathrm{TeV}$ at $95 \%$ C.L. on the extinction mass scale (equivalent to the fundamental Planck scale) [37].

Lower bounds on the Planck scale can also be derived by non-observation of production and decay of $\mathrm{TeV} \mathrm{BHs}$ in collider experiments and cosmic ray observations [38-45]. The CMS and ATLAS (A Toroidal LHC ApparatuS) collaborations have conducted searches for BH signatures at the LHC [13, 46, 47], setting limits on the production cross section and the minimal $\mathrm{BH}$ mass $M_{\text {min }}$, i.e., the minimum mass at which a $\mathrm{BH}$ can form. Depending on model assumptions and using different final states, the CMS collaboration excludes $M_{\text {min }}$ below 4.3 to $6.2 \mathrm{TeV}$, while the ATLAS collaboration excludes $M_{\text {min }}$ below 4.8 to 6.2 TeV [46] and 4.6 to $6.2 \mathrm{TeV}$ [47], all at 95\% C.L.. The recent $13 \mathrm{TeV}$ analysis done by the ATLAS collaboration has excluded the production of a rotating black hole with $n=6[48]$.

The purpose of this paper is to revisit and extend the above results from CMS. We simulate production and decay of microscopic BHs at the LHC with the Monte Carlo generator CATFISH (v2.10) [14]. We then derive experimental bounds on the fundamental Planck 
mass and the number of extra dimensions by comparing simulation results with modelindependent experimental limits on BH production from the CMS Collaboration [13]. The absence of observed black hole events in the CMS experimental data allows us to set bounds on various physical parameters of the ADD model and to constrain the minimum mass of $\mathrm{TeV}$-scale black holes which may form in hadronic scattering processes.

\section{Black hole formation in particle collisions}

According to the Hoop Conjecture [49], a BH forms when a mass $M$ is confined to a region of typical size equal to the Schwarzschild radius for that mass, $R_{\mathrm{S}}(M)$. Therefore, if two particles collide with center of mass energy $\sqrt{s}$ and impact parameter smaller than $R_{\mathrm{S}}(\sqrt{s})$, a $\mathrm{BH}$ may form. If $R_{\mathrm{S}} \ll R$, as expected in the ADD scenario, the newly formed BH lives in a $D$-dimensional space-time with negligible curvature at the $\mathrm{BH}$ scale. In this case, the Schwarzschild radius of the $\mathrm{BH}$ can be expressed as [50-52],

$$
R_{\mathrm{S}}=\frac{1}{\sqrt{\pi} M_{*}}\left[\frac{8 \Gamma\left(\frac{n+3}{2}\right)}{n+2}\right]^{\frac{1}{n+1}}\left(\frac{M}{M_{*}}\right)^{\frac{1}{n+1}},
$$

where $M=(1-y) \sqrt{s}$, and $y$ is the fraction of energy which escapes into the bulk as gravitons, depending on the impact parameter. The Hoop Conjecture implies a $\mathrm{BH}$ production cross section $\sigma(s, n, y)=\pi F R_{\mathrm{S}}^{2}$, where the form factor $F \leq 1$ is related to $y$ and accounts for the energy of the colliding particles which is not trapped in the event horizon, the so called "graviton energy loss at formation." Since BH production in hadron colliders occurs at the parton level, the total cross section for a hadronic collision is obtained by integrating over the Parton Distribution Functions (PDFs) of the hadrons [51]

$$
\sigma_{p p \rightarrow \mathrm{BH}}(s, n, y)=\sum_{i j} \int_{0}^{1} 2 z \mathrm{~d} z \int_{x_{m}}^{1} \mathrm{~d} x \int_{x}^{1} \frac{\mathrm{d} x^{\prime}}{x^{\prime}} f_{i}\left(x^{\prime}, Q\right) f_{j}\left(x / x^{\prime}, Q\right) \sigma(\sqrt{x s}, n, y),
$$

where $f_{i}(x, Q)$ are the PDFs with four-momentum transfer squared $Q$, and $z$ is the impact parameter normalized to its maximum value. The cutoff at small $x$ is $x_{m}=M_{\min }{ }^{2} /\{s[(1-$ $\left.y(z)]^{2}\right\}$, where $M_{\min }$ is the minimum-allowed mass of the BH. The total cross section in the absence of graviton energy loss at formation is recovered by setting $F=1$ (Black Disk (BD) cross section) [14].

If the initial $\mathrm{BH}$ mass is much larger than the Planck mass, a semiclassical treatment suggests that the newly-formed $\mathrm{BH}$ decays through four, possibly distinct stages: balding, spin-down, thermal evaporation and quantum decay [53]. During the balding stage, the $\mathrm{BH}$ radiates multipole momenta and quantum numbers [10,54], eventually settling down to a $D$-dimensional Kerr geometry. Angular momentum is radiated during the spindown stage [54]. The Schwarzschild BH then decays into elementary particles through the Hawking mechanism (thermal evaporation stage). Most of the energy of the $\mathrm{BH}$ is radiated in this stage, with SM particles dominating the decay products. When the mass of the evaporating $\mathrm{BH}$ approaches the Planck scale, $Q_{\min } \sim M_{*}$, the $\mathrm{BH}$ enters the quantum phase, where the decay ceases to be semiclassical and becomes dominated by quantum gravitational effects. 


\section{$3 \quad \mathrm{BH}$ event simulations}

Several Monte Carlo generators for $\mathrm{BH}$ production at colliders have been developed over the years: TRUENOIR [55], CHARYBDIS2 [56, 57], BlackMax [58, 59] (used by CMS), QBH [60] and an unnamed generator by Tanaka et al. [61]. Our analysis is based on CATFISH (Collider grAviTational FIeld Simulator for black Holes) [14]. CATFISH is a Fortran 77 Monte Carlo generator designed specifically for simulating BH events at CERN's LHC. It incorporates three models for BH formation and cross section: BD, Yoshino-Nambu (YN) Trapped Surface (TS) [62], and Yoshino-Rychkov (YR) improved TS model [63]. The lack of a quantum theory of gravity requires a phenomenological treatment of the final stage. CATFISH offers the choice of simulating the quantum phase by either nonthermally decaying the $\mathrm{BH}$ into a number $n_{p}$ of hard quanta, each with energy $Q_{\min } / n_{p}$, or forming a BH remnant. CATFISH also incorporates several other physical effects, such as inelasticity, exact field emissivities and corrections to semiclassical BH evaporation. The generator interfaces to the PYTHIA Monte Carlo fragmentation code [64] using the Les Houches interface [65]. In our analysis, we run CATFISH (v2.10) with the CTEQ6PDF PDF set and PYTHIA (v6.425) Tune Z1.

The simulation of a BH event in CATFISH follows these steps. First, CATFISH computes the total and differential cross sections for the $\mathrm{BH}$ formation. The initial $\mathrm{BH}$ mass is sampled from the differential cross section. The $\mathrm{BH}$ is then decayed through the Hawking mechanism until the $\mathrm{BH}$ mass reaches the quantum limit, where a final nonthermal hard event is generated or a $\mathrm{BH}$ remnant is created. The unstable quanta emitted by the $\mathrm{BH}$ are instantaneously hadronized or decayed by PYTHIA, which also simulates initial- and final-state radiation particles. To determine the physics of $\mathrm{BH}$ formation and decay, CATFISH uses several external parameters and switches:

- $A D D$ parameters

1. Fundamental Planck mass: $\operatorname{MSTAR}=M_{*}$.

2. Number of extra dimensions: $\operatorname{NEXTRADIM}=n, \operatorname{NEXTRADIM}=3,4,5,6$.

- BH formation parameters

1. Graviton energy loss at formation: GRAVITONLOSS $=0$ (BD model), 1 (YR or YN TS models, see below).

2. Gravitational loss model: GRAVITONMODEL $=0$ (YN TS model [62]), 1 (YR improved TS model [63]).

3. Minimum initial BH mass in Planck units: $\mathrm{XMIN}=M_{\min } / M_{*}, \mathrm{XMIN} \geq 1$.

- BH evaporation parameters

1. Minimum quantum BH mass in Planck units: QMIN $=Q_{\min } / M_{*}$, QMIN $\leq$ XMIN.

2. Number of quanta emitted in the Planck phase: $\mathrm{NP}=n_{p}$. When $\mathrm{NP}=0$, the $\mathrm{BH}$ forms a stable remnant with mass $Q_{\min }$. 
The primary goal of this investigation is to determine lower bounds on the $D$ dimensional fundamental Planck scale for different values of NEXTRADIM, XMIN and NP using the model-independent 95\% C.L. upper limits on the BH cross section from the CMS search [13]. We also derive lower limits on $M_{\min }$ and XMIN for fixed MSTAR, NEXTRADIM and NP. For simplicity, we consider only BH formation with GRAVITONLOSS=0 (BD cross section), XMIN=QMIN and final decay into 2, 4, or 6 quanta or formation of a stable $\mathrm{BH}$ remnant $(\mathrm{NP}=0)$. The stable $\mathrm{BH}$ remnant is invisible to the detector and thus contributes to missing energy. The results with GRAVITONLOSS $=1$ will be presented in a future report.

The CMS search for BH events looks at excess transverse energy with respect to SM background predictions [13]. The transverse energy $S_{\mathrm{T}}$ of an event is defined as the scalar sum of the transverse energies of all the final-state objects in excess of $50 \mathrm{GeV}$, i.e., jets, muons, electrons and photons satisfying the selection criteria discussed in ref. [13]. The missing transverse energy is defined as the magnitude of the vector sum of the transverse momenta of all the final-state objects. If it is greater than $50 \mathrm{GeV}$, the missing transverse energy is added to $S_{\mathrm{T}}$. The event multiplicity $N$ is defined as the number of final-state objects which are used to calculate $S_{\mathrm{T}}$. BH events are expected to have high multiplicities.

\section{Results}

Lower bounds on $M_{*}$ and $M_{\min }$ are derived by evaluating the partial cross section $\sigma\left(S_{\mathrm{T}}>\right.$ $S_{\mathrm{T}}^{\min }$ ) for events whose $S_{\mathrm{T}}>S_{\mathrm{T}}^{\min }$ and whose multiplicities are greater than some chosen value, given by,

$$
\sigma\left(S_{\mathrm{T}}>S_{\mathrm{T}}^{\min }\right)=k \cdot \sigma_{p p \rightarrow \mathrm{BH}},
$$

where $S_{T}^{\min }$ is the minimal transverse energy chosen, and $k$ is

$$
k=\frac{\text { Num. of events with } S_{\mathrm{T}}>S_{\mathrm{T}}^{\min }}{\text { Total Num. of events }} .
$$

The behavior of the total cross section $\sigma_{p p \rightarrow \mathrm{BH}}$ as a function of $M_{*}$ and $M_{\min }$ (i.e., XMIN) follows from eqs. (2.1), (2.2). There are 3 factors: 1) The BD cross section $\sigma_{\mathrm{BD}}$ is inversely proportional to a power of $M_{*} ; 2$ ) Since XMIN appears as a lower limit of integration in the total cross section $\sigma_{p p \rightarrow \mathrm{BH}}$, the greater XMIN, the smaller $\sigma_{p p \rightarrow \mathrm{BH}}$ is at a fixed $\left.M_{*} ; 3\right) \mathrm{In}$ addition, the PDFs fall off rapidly at high $Q$. Taking into account all these factors, $\sigma_{p p \rightarrow \mathrm{BH}}$ is expected to decrease as $M_{*}$ (XMIN) increases at fixed XMIN $\left(M_{*}\right)$. The ratio $k$ in eq. (4.2) can be estimated by integrating over the spectra of visible final state particles over the range $S_{\mathrm{T}}>S_{\mathrm{T}}^{\mathrm{min}}$ and then averaging over all final state particles. $k$ is an increasing function of the Hawking temperature, which, in turn, is a monotonically increasing function of $M_{*}$. Thus, as $M_{*}$ increases, the graph of $k$ vs. $S_{\mathrm{T}}^{\min }$ flattens. Following the CMS collaboration [66], we choose the signal acceptance to be $100 \%$. In summary, $\sigma\left(S_{\mathrm{T}}>S_{\mathrm{T}}^{\min }\right) \times A$ is expected to decrease as either $M_{*}$ or XMIN increases.

The partial cross section $\sigma\left(S_{\mathrm{T}}>S_{\mathrm{T}}^{\min }\right)$ as a function of $M_{*}$ is obtained by running CATFISH with fixed NEXTRADIM, GRAVITONLOSS, XMIN $=$ QMIN, NP. The results are shown by the upper two graphs in figure 1 for NEXTRADIM $=3$, XMIN $=\mathrm{QMIN}=5, \mathrm{NP}=0,4$. The lower two graphs in figure 1 display $\sigma\left(S_{\mathrm{T}}>S_{\mathrm{T}}^{\min }\right)$ as a function of XMIN for $M_{*}=1.5 \mathrm{TeV}$, 

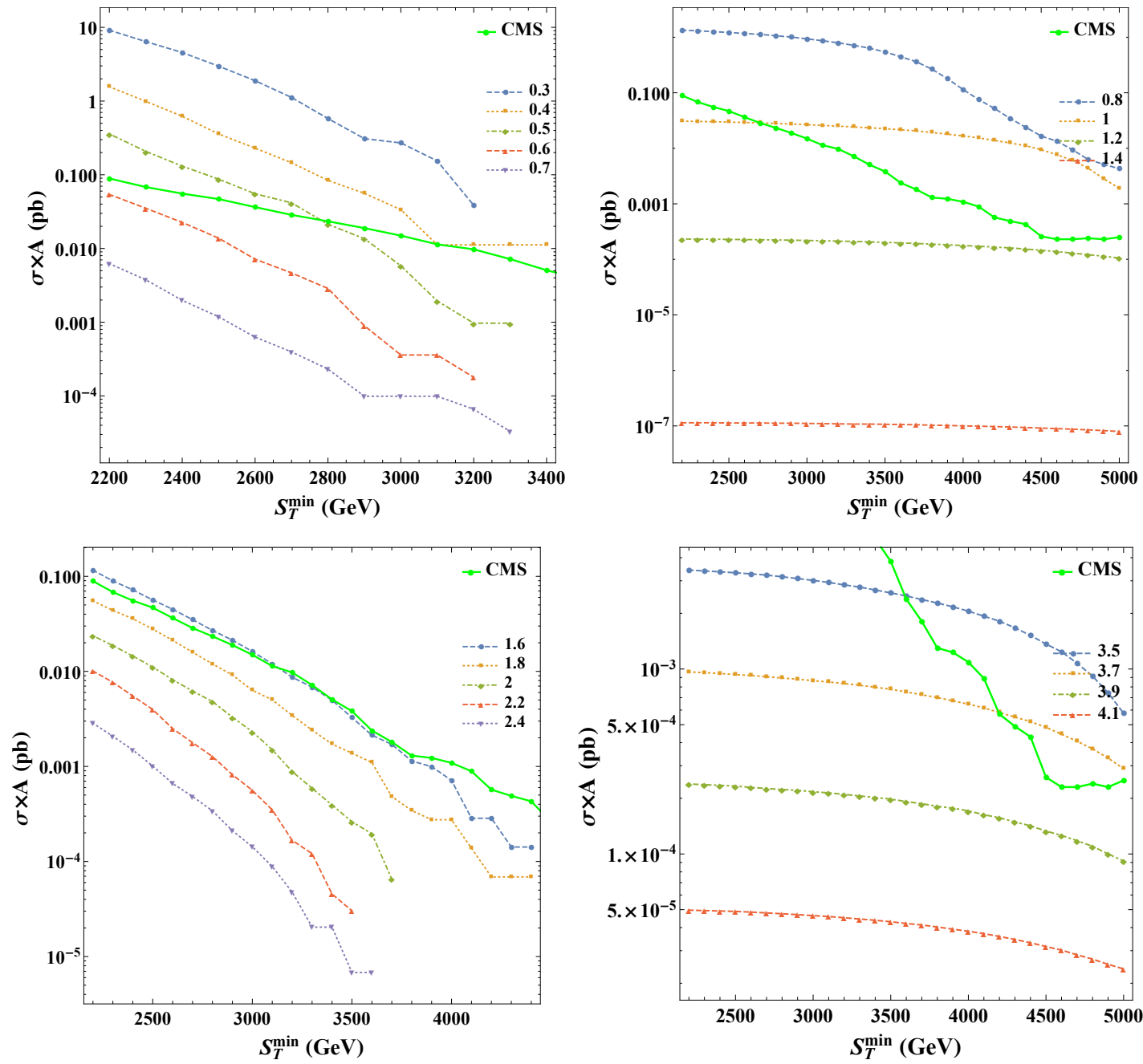

Figure 1. $\sigma\left(S_{\mathrm{T}}>S_{\mathrm{T}}^{\min }\right) \times A$ as a function of $M_{*}$ (Upper two graphs, numbers being $M_{*}$ 's chosen in units of TeV) or XMIN (Lower two graphs, numbers being XMIN's) at NP $=0$ (Right two graphs) and $\mathrm{NP}=4$ (Left two graphs). The model-independent 95\% CL experimental upper limits for counting experiments from the CMS Collaboration are also shown. The multiplicity is $N \geq 3$.

$\operatorname{NEXTRADIM}=5$, and NP $=0,4$. As expected, figure 1 shows that the cross section decreases as $M_{*}$ and XMIN increase.

Figure 1 can be used to determine bounds on $M_{*}$ and XMIN by comparing the simulated $\sigma\left(S_{\mathrm{T}}>S_{\mathrm{T}}^{\mathrm{min}}\right)$ with the experimental limits (the solid curves in the graphs). For example, the upper right plot shows that the lower limit on $M_{*}$ lies in the range $1.0 \mathrm{TeV}-1.2 \mathrm{TeV}$, and the bottom right plot shows that the lower limit on XMIN lies in the range 3.7-3.9. Multiplying this range by $M_{*}=1.5 \mathrm{TeV}$, we obtain the lower limits on $M_{\min }=5.55 \mathrm{TeV}-5.85 \mathrm{TeV}$.

We run CATFISH over a large range of parameter space (see appendix A) and require the simulated $\sigma\left(S_{\mathrm{T}}>S_{\mathrm{T}}^{\min }\right)$ to be less than the experimental limits for all muliplicities $N \geq 3,4, \ldots, 10$. Figure 2 shows the exclusion region for $M_{*}$. As expected, the lower limit $M_{*, \exp }$ is a decreasing function of XMIN. The value of $M_{* \text {,exp }}$ does not strongly depend on 

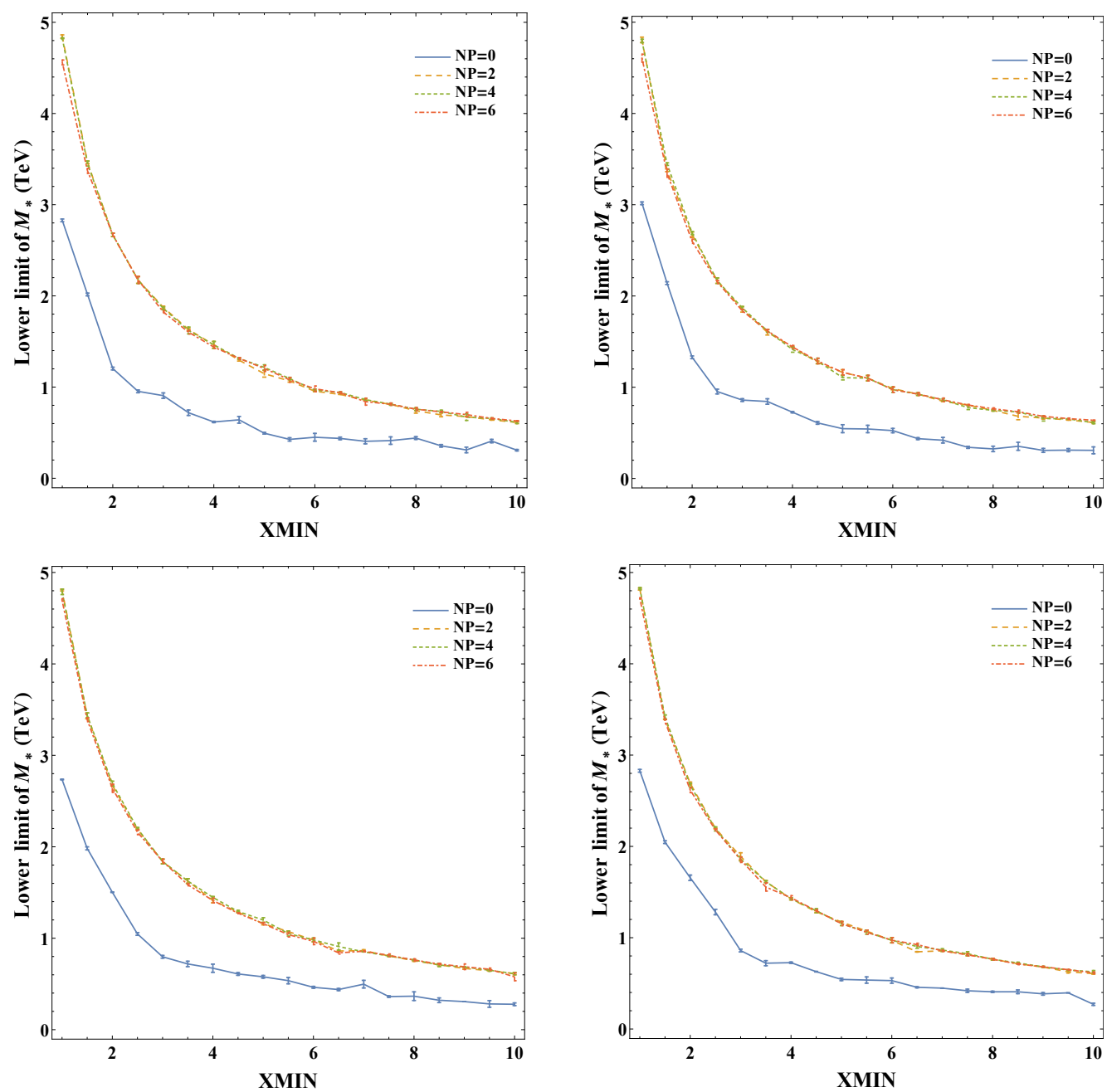

Figure 2. Simulated lower limit on $M_{*}$ vs. XMIN as a function of NP and NEXTRADIM $=3$ (top left), 4 (top right), 5 (bottom left), and 6 (bottom right).

NP, as long as NP $\neq 0$. If NP $=0$, the bound on $M_{* \text {, exp }}$ becomes much smaller. This is due to the high transverse momentum of the $\mathrm{BH}$ remnant, which contributes to the missing energy. The lower limits on $M_{*}$ set upper bounds on $M_{\min }$ (XMIN). As experimental data exclude values of $M_{*} \lesssim 1 \mathrm{TeV}$ [14], our results for NP $=0$ set an upper limit XMIN $\lesssim 2.5$. NP $\neq 0$ results give the milder constraint, XMIN $\lesssim 6$. More experimental limits are shown in table 1, leading to more stringent constraints. For instance, the CMS searches for events with an energetic jet and an imbalance in transverse momentum at $\sqrt{s}=8 \mathrm{TeV}[26]$ have set the lower limit $M_{*} \sim 2.71 \mathrm{TeV}$ for $n=3$. The upper left panel in figure 2 shows that the events with microscopic BHs decaying to remnants $(\mathrm{NP}=0)$ are excluded, and the experimentally allowed range of XMIN is restricted to $1 \sim 2$ for NP $\neq 0$. The lower limits of $M_{*}$ for $n=4,5,6$ do not exclude events with remnants as BH final products, but never the less, set strong constraints on the ranges where the semi-classical treatment is valid.

Figure 3 shows the exclusion region for XMIN. As expected, the lower limit of XMIN is a decreasing function of $M_{*}$. Figure 3 also shows that these limits do not depend on NP 

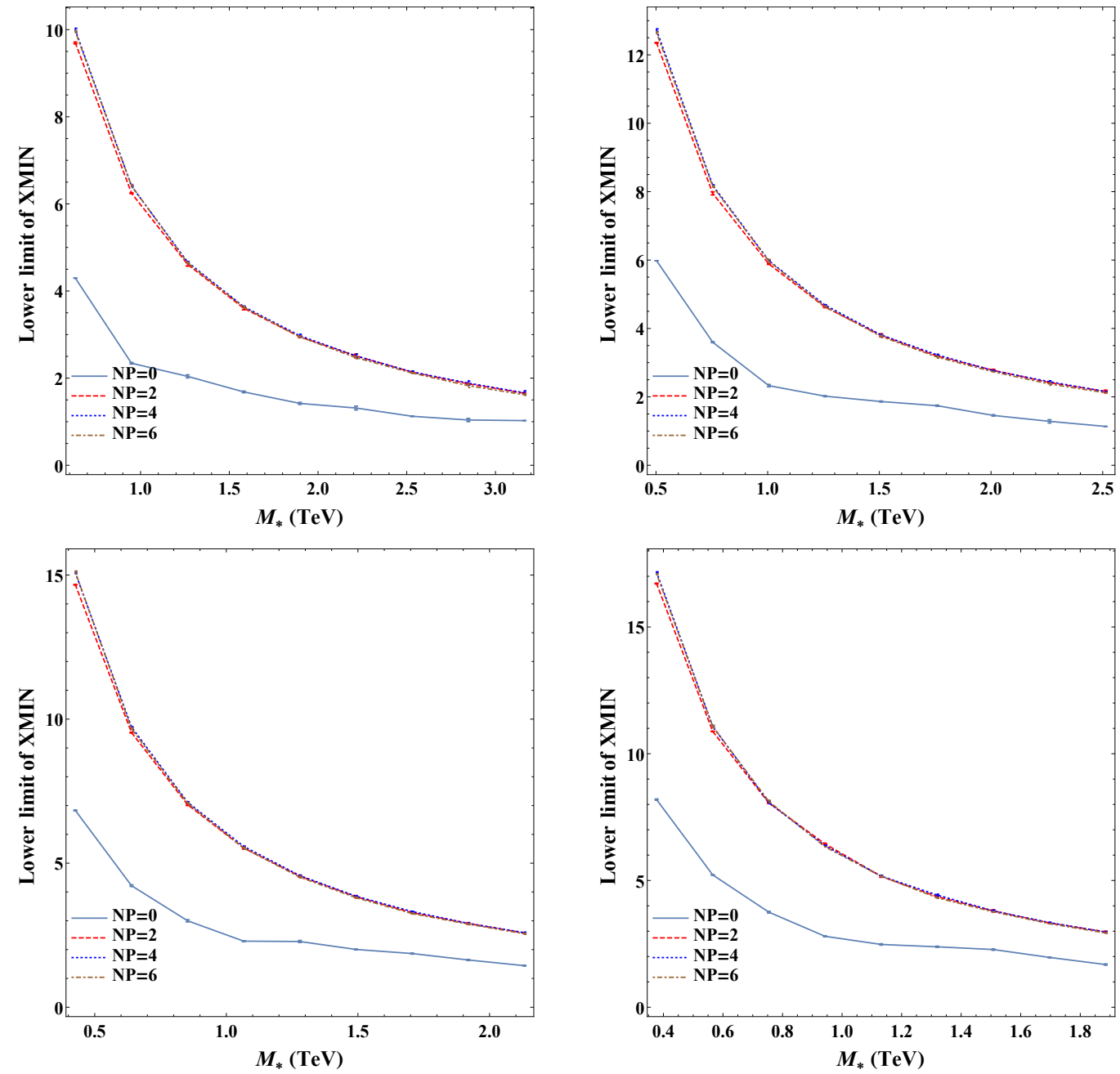

Figure 3. Simulated lower limit on XMIN vs. $M_{*}$ as a function of NP and NEXTRADIM $=3$ (top left), 4 (top right), 5 (bottom left), and 6 (bottom right).

strongly when NP $\neq 0$, but become much smaller at NP $=0$. This figure can be combined with figure 2 to constrain $M_{*}$ further. For example, if there are 3 extra dimensions, and a $\mathrm{BH}$ decays into 2 quanta in the quantum phase, the dashed curve (NP $=2)$ in the upper left plot shows that XMIN $\gtrsim 4$ at $M_{*} \sim 1.5 \mathrm{TeV}$. At the same time, the dashed curve in the upper left plot $(n=3)$ of figure 2 indicates that if XMIN $=4, M_{*} \gtrsim 1.5 \mathrm{TeV}$. This shows that the lower limits of $M_{*}$ are consistent with those of XMIN. In figure 3, the ranges of $M_{*}$ were chosen in order to compare the CATFISH results with those of BlackMax and CHARYBDIS2 from ref.'s [13, 46].

Figure 4 compares the lower limits of $M_{\text {min }}$ predicted by CATFISH with those from BlackMax and CHARYBDIS2 done by the CMS Collaboration [13]. It shows that as long as NP $=0$, CATFISH's limits are much smaller than those of BlackMax and CHARYBDIS2. The difference in predictions between CATFISH and CHARYBDIS2 when a stable remnant model is produced is due to the different treatments of the quantum phase by the two 

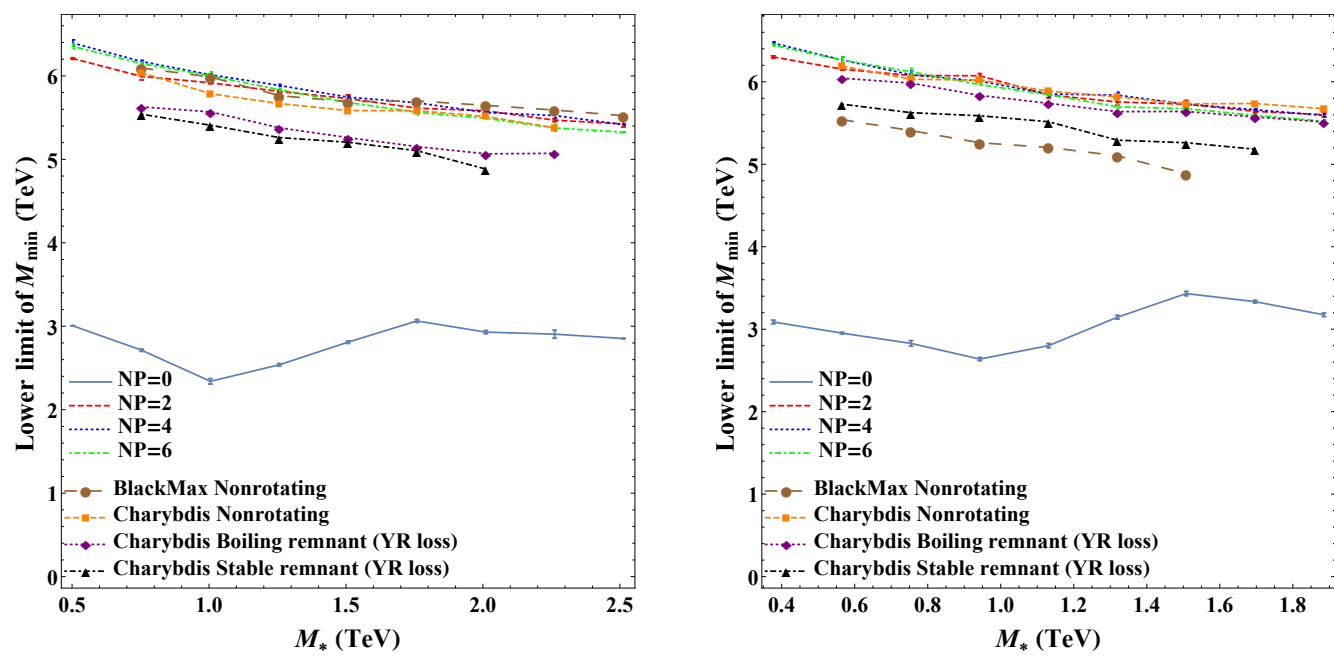

Figure 4. Comparison of the predictions on lower limits of $M_{\mathrm{BH}}^{\min }$ from CATFISH with those from BlackMax and CHARYBDIS2 at NEXTRADIM $=4$ (left) and 6 (right). The results of BlackMax and CHARBDIS are extracted from figure 4 in ref. [13].

generators $[13,67,68]$. Moreover, CATFISH's stable remnant is invisible to the detector and contributes to missing energy, while CHARYBDIS2's remnant behaves as a heavy fundamental particle with conventional interactions in the detector. If NP $\neq 0$, the situation is more complicated. CATFISH agrees with BlackMax (nonrotating BH model) very well when $n=4$, but gives higher limits than BlackMax if $n=6$. CATFISH gives limits similiar to those of CHARYBDIS2 (nonrotating BH model) for $n=4,6$. Figure 5 shows the comparison between CATFISH predictions with those from the ATLAS Collaboration [46]. CATFISH agrees with BlackMax and CHARYBDIS2 when NP $\neq 0$, but predicts much smaller limits when NP $=0$. The similarities among the three generators as shown in the two figures for $M_{\min }$ vs $M_{*}$ for NP $\neq 0$ are due to the fact that the three generators incorporate the same basic physics of microscopic $\mathrm{BH}$ formation and decay. However, the three generators differ from one another in the implementation of the quantum phase and in the inclusion or exclusion of the effect of gravitational energy loss at the formation of the BHs. For example, the predictions of the behavior of $M_{\min }$ vs. $M_{*}$ for NP $=0$ (BH remnant) by CATFISH differ from those of BlackMax and CHARYBDIS2.

\section{Conclusions}

In this work, lower limits on the fundamental Planck scale $M_{*}$ and minimal BH mass $M_{\text {min }}$ at formation have been obtained in a vast parameter space, using experimental upper limits on the partial production cross section of microscopic BHs [13]. Various models implemented in CATFISH have been explored and different limits have been determined. Our results for models without a BH stable remnant generally agree with earlier results by the CMS collaboration and the ATLAS collaboration based on the BlackMax and CHARYBDIS generators, where some of the observed discrepancies can be explained by the different methods used by the analyses to constrain $M_{D}$. The dedicated CMS limits use the optimum 

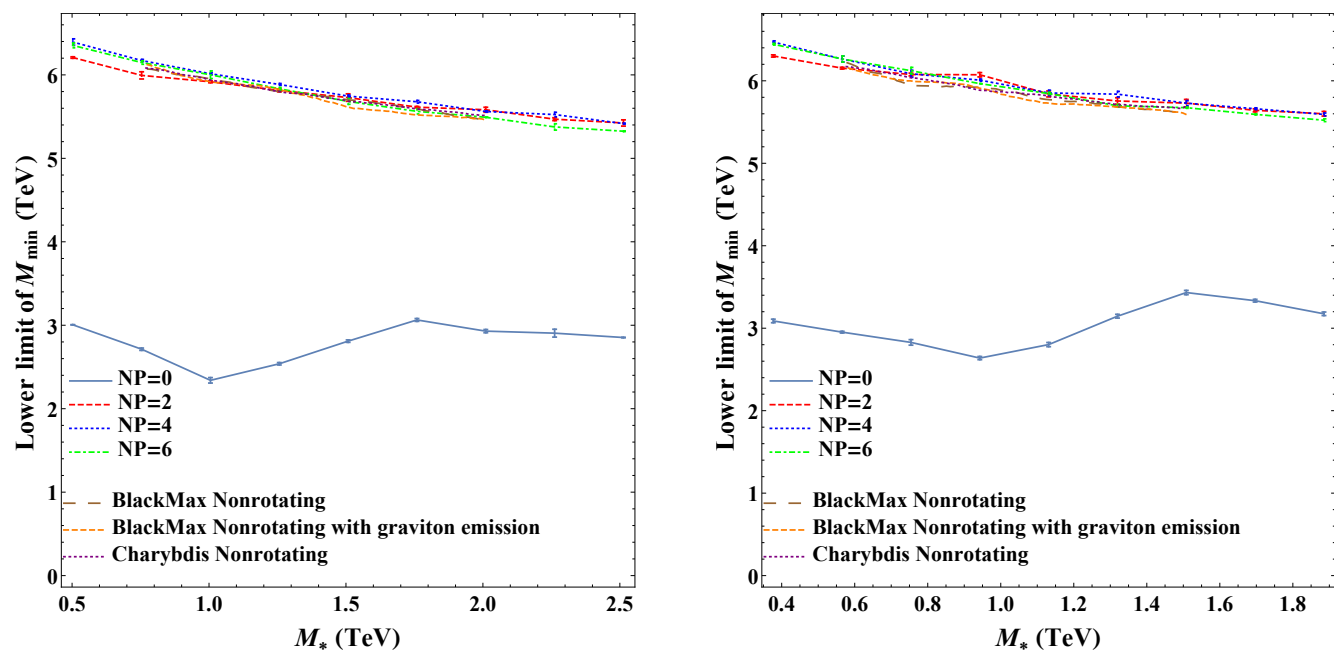

Figure 5. Comparison of the predictions on lower limits of $M_{\mathrm{BH}}^{\min }$ from CATFISH with those from BlackMax and CHARYBDIS2 at NEXTRADIM $=4$ (left) and 6 (right). The results of BlackMax and CHARBDIS are extracted from figure's 8 and 10 in ref. [46].

minimum multiplicity distribution for each case considered, while we require the simulated cross section to be less than the CMS limits for all multiplicities $\geq 3, \ldots, 10$. In general, $\mathrm{BH}$ remnant models give milder constraints than non-remnant models. The calculated lower bounds on $M_{*}$ constrain the the size of LEDs in ADD models. Future investigations will focus on performing a similar analysis to the one carried out in the present paper with the additional feature of graviton energy loss during BH formation (GRAVITONLOSS $=1$ ). Another refinement of the models is to include the effects of the generalized uncertainty principle [71, 72]. The steps outlined above for the simulation of microscopic $\mathrm{BH}$ events can also be carried out for string balls, string resonances and other exotic particles.

\section{Acknowledgments}

We wish to thank C. Henderson and P. Rumerio for their constructive discussions. We also wish to thank G. Landsberg and the CMS Exotica conveners for their help. We gratefully acknowledge RC2 [73] at the University of Alabama and the Alabama Supercomputer Authority [74] for providing the computing infrastructure essential to our analyses.

\section{A Procedure for determining $M_{*}$ and $M_{\min }$ limits}

This appendix describes how to determine the limits on the $D$-dimensional fundamental Planck scale $M_{*}$, or equivalently, the reduced Planck scale $M_{D}$ (eq. (1.1)) used in ref. [13], for different NEXTRADIM $(=3,4,5,6)$, GRAVITONLOSS $(=0)$, XMIN (= QMIN) and NP $(=0,2$, $4,6)$. The model-independent $95 \%$ C.L. upper cross section limits for counting experiments (figures 6 and 7 in ref. [13]) were used. CATFISH accepts only XMIN $\geq 1$, so the lower limit of XMIN is 1 and its upper limit is determined by noticing that the mass of the BH must 
be less than the center of mass energy of the LHC,

$$
\mathrm{XMIN} \times \operatorname{MSTAR} \leq 8 \mathrm{TeV} .
$$

The stepsize of XMIN is set to 0.5. We use a bisection method to find the limits on the $D$-dimensional fundamental Planck scale:

Step 1. The possible range of $M_{*}$ is determined by fixing all other parameters. The lower limit is $m_{0}=1 \mathrm{TeV}$ and the upper limit, $M_{0}$, satisfies eq. (A.1) and the condition

$$
\sigma \cdot L \geq \frac{1}{N_{\text {run }}}
$$

where $\sigma$ is the cross section of the production, $L=12 \mathrm{fb}^{-1}$ is the LHC integrated luminosity, and $N_{\text {run }}$ is the number of events of each run. In our simulations we choose $N_{\text {run }}=10^{4}$.

Step 2. CATFISH is run with $\operatorname{MSTAR}=M_{1}=\left(m_{0}+M_{0}\right) / 2$ and the simulated partial cross section $\sigma\left(S_{\mathrm{T}}>S_{\mathrm{T}}^{\min }\right)$ is compared with CMS cross section limits [13]. We require that the simulated $\sigma\left(S_{\mathrm{T}}>S_{\mathrm{T}}^{\min }\right)$ be less than CMS limits for all multiplicities $(\geq 3,4, \ldots, 10)$.

Step 3. If $M_{1}$ is allowed by experimental data, i.e., the simulated cross section (times the detector acceptance $A$ ) for $\mathrm{BH}$ production is too small, $M_{*}$ must be smaller than $M_{1}$. CATFISH is run with $\operatorname{MSTAR}=M_{2}=\left(m_{0}+M_{1}\right) / 2$. Otherwise, CATFISH is run with $\operatorname{MSTAR}=M_{2}=\left(M_{1}+M_{0}\right) / 2$.

Step 4. Step 3 is repeated $i$ times until $\left|M_{i}-M_{i-1}\right|<\Delta M$, where $M_{i}$ is the result of the $i$-th simulation and $\Delta M$ is the required precision, $\Delta M=0.1 \mathrm{TeV}$.

Step 5. The more likely value of $M_{*}$ is then determined as

$$
M_{*, \exp }=\frac{M_{i}+M_{i-1}}{2} .
$$

The error on $M_{*, \exp }$ is $\Delta M_{*, \exp }=p\left|M_{i}-M_{i-1}\right|$, where $p$ is determined at a given confidence level (C.L.) for the interval $\left[\operatorname{Min}\left(M_{i}, M_{i-1}\right), \operatorname{Max}\left(M_{i}, M_{i-1}\right)\right]$,

$$
p=\frac{1-\text { C.L. } / 100}{2} \text {. }
$$

The lower limit on the Planck mass, $M_{* \text {,exp }}$, is determined for a given choice of NEXTRADIM, GRAVITONLOSS, XMIN (= QMIN) and NP. Different sets of these parameters are chosen and Steps 1-5 are repeated to determine $M_{*, \exp }$ as a function of the parameters. Similarily, the lower limit of $M_{\text {min }}$ or XMIN can be obtained for different choices of MSTAR, NEXTRADIM, GRAVITONLOSS $(=0)$ and NP.

Open Access. This article is distributed under the terms of the Creative Commons Attribution License (CC-BY 4.0), which permits any use, distribution and reproduction in any medium, provided the original author(s) and source are credited.

\section{References}

[1] E.A. Mirabelli, M. Perelstein and M.E. Peskin, Collider signatures of new large space dimensions, Phys. Rev. Lett. 82 (1999) 2236 [hep-ph/9811337] [INSPIRE]. 
[2] N. Arkani-Hamed, S. Dimopoulos and G.R. Dvali, The hierarchy problem and new dimensions at a millimeter, Phys. Lett. B 429 (1998) 263 [hep-ph/9803315] [INSPIRE].

[3] I. Antoniadis, N. Arkani-Hamed, S. Dimopoulos and G.R. Dvali, New dimensions at a millimeter to a Fermi and superstrings at a TeV, Phys. Lett. B 436 (1998) 257 [hep-ph/9804398] [INSPIRE].

[4] N. Arkani-Hamed, S. Dimopoulos and G.R. Dvali, Phenomenology, astrophysics and cosmology of theories with submillimeter dimensions and TeV scale quantum gravity, Phys. Rev. D 59 (1999) 086004 [hep-ph/9807344] [INSPIRE].

[5] T. Han, J.D. Lykken and R.-J. Zhang, On Kaluza-Klein states from large extra dimensions, Phys. Rev. D 59 (1999) 105006 [hep-ph/9811350] [INSPIRE].

[6] J.L. Hewett, Indirect collider signals for extra dimensions, Phys. Rev. Lett. 82 (1999) 4765 [hep-ph/9811356] [INSPIRE].

[7] P.C. Argyres, S. Dimopoulos and J. March-Russell, Black holes and submillimeter dimensions, Phys. Lett. B 441 (1998) 96 [hep-th/9808138] [INSPIRE].

[8] T. Banks and W. Fischler, A model for high-energy scattering in quantum gravity, hep-th/9906038 [INSPIRE].

[9] G.F. Giudice, R. Rattazzi and J.D. Wells, Quantum gravity and extra dimensions at high-energy colliders, Nucl. Phys. B 544 (1999) 3 [hep-ph/9811291] [INSPIRE].

[10] S.B. Giddings and S.D. Thomas, High-energy colliders as black hole factories: the end of short distance physics, Phys. Rev. D 65 (2002) 056010 [hep-ph/0106219] [INSPIRE].

[11] S. Dimopoulos and G.L. Landsberg, Black holes at the LHC, Phys. Rev. Lett. 87 (2001) 161602 [hep-ph/0106295] [INSPIRE].

[12] K. Cheung, Black hole, string ball and p-brane production at hadronic supercolliders, Phys. Rev. D 66 (2002) 036007 [hep-ph/0205033] [INSPIRE].

[13] CMS collaboration, Search for microscopic black holes at $\sqrt{s}=8 \mathrm{TeV}$ with the CMS detector, JHEP 07 (2013) 178 [arXiv: 1303.5338] [CMS-PAS-EXO-12-009].

[14] M. Cavaglia, R. Godang, L. Cremaldi and D. Summers, Catfish: a Monte Carlo simulator for black holes at the LHC, Comput. Phys. Commun. 177 (2007) 506 [hep-ph/0609001] [INSPIRE].

[15] FERMI-LAT collaboration, Limits on large extra dimensions based on observations of neutron stars with the Fermi-LAT, JCAP 02 (2012) 012.

[16] C. Hanhart, J.A. Pons, D.R. Phillips and S. Reddy, The likelihood of GODs' existence: improving the SN1987 a constraint on the size of large compact dimensions, Phys. Lett. B 509 (2001) 1 [astro-ph/0102063] [INSPIRE].

[17] S. Hannestad and G.G. Raffelt, Supernova and neutron star limits on large extra dimensions reexamined, Phys. Rev. D 67 (2003) 125008 [Erratum ibid. D 69 (2004) 029901] [hep-ph/0304029] [INSPIRE].

[18] T. Gleisberg et al., Helicity formalism for spin-2 particles, JHEP 09 (2003) 001.

[19] LEP Exotica Working Group, Combination of LEP results on direct searches for large extra dimensions, report 2004-03 (2004). 
[20] CDF collaboration, T. Aaltonen et al., Search for large extra dimensions in final states containing one photon or jet and large missing transverse energy produced in $\bar{p} \bar{p}$ collisions at $\sqrt{s}=1.96$ TeV, Phys. Rev. Lett. 101 (2008) 181602 [arXiv:0807.3132] [INSPIRE].

[21] D0 collaboration, V.M. Abazov et al., Search for large extra dimensions via single photon plus missing energy final states at $\sqrt{s}=1.96$ TeV, Phys. Rev. Lett. 101 (2008) 011601 [arXiv:0803.2137] [INSPIRE].

[22] D0 collaboration, V.M. Abazov et al., Search for large extra dimensions in the monojet + missing $E_{T}$ channel at DO, Phys. Rev. Lett. 90 (2003) 251802 [hep-ex/0302014] [INSPIRE].

[23] CMS collaboration, Search for new physics with a monojet and missing transverse energy in pp collisions at $\sqrt{s}=7$ TeV with 1.1 $\mathrm{fb}^{-1}$, CMS-PAS-EXO-11-059 (2011) [arXiv:1206.5663].

[24] CMS collaboration, Search for extra dimensions using the monophoton final state, CMS-PAS-EXO-11-058 (2011).

[25] ATLAS collaboration, Search for new phenomena in monojet plus missing transverse momentum final states using $10 \mathrm{fb}^{-1}$ of pp collisions at $\sqrt{\mathrm{s}}=8$ TeV with the ATLAS detector at the LHC, ATLAS-CONF-2012-147 (2012).

[26] CMS collaboration, Search for new physics in monojet events in pp collisions at $\sqrt{s}=8$ TeV, CMS-PAS-EXO-12-048 (2012).

[27] CMS collaboration, Search for large extra spatial dimensions in dielectron production with the CMS detector, CMS-PAS-EXO-12-031 (2012).

[28] CMS collaboration, Search for large extra dimensions in dimuon events in pp collisions at $\sqrt{s}=8 \mathrm{TeV}$, CMS-PAS-EXO-12-027 (2012).

[29] CMS collaboration, Search for quark contact interactions and extra spatial dimensions using dijet angular distributions in proton-proton collisions at $\sqrt{s}=8 \mathrm{TeV}$, Phys. Lett. $\mathbf{B} 746$ (2015) 79 [arXiv:1411.2646] [INSPIRE].

[30] CMS collaboration, Search for physics beyond the standard model in dilepton mass spectra in proton-proton collisions at $\sqrt{s}=8 \mathrm{TeV}$, JHEP 04 (2015) 025.

[31] CMS collaboration, Search for dark matter, extra dimensions, and unparticles in monojet events in proton-proton collisions at $\sqrt{s}=8$ TeV, Eur. Phys. J. C 75 (2015) 235.

[32] ATLAS collaboration, Search for new phenomena in final states with an energetic jet and large missing transverse momentum in pp collisions at $\sqrt{s}=8 \mathrm{TeV}$ with the ATLAS detector, Eur. Phys. J. C 75 (2015) 299 [arXiv:1502.01518] [INSPIRE].

[33] ATLAS collaboration, Search for contact interactions and large extra dimensions in the dilepton channel using proton-proton collisions at $\sqrt{s}=8 \mathrm{TeV}$ with the ATLAS detector, Eur. Phys. J. C 74 (2014) 3134 [arXiv:1407.2410] [INSPIRE].

[34] CMS collaboration, $Z^{\prime} \rightarrow \tau \tau-e \mu$ final state, CMS-PAS-EXO-12-046 (2012).

[35] CMS collaboration, Search for new phenomena in monophoton final states in proton-proton collisions at $\sqrt{s}=8 \mathrm{Te}$, PAS-EXO-12-047 [arXiv:1410.8812].

[36] ATLAS collaboration, Search for new phenomena in events with a photon and missing transverse momentum in pp collisions at $\sqrt{s}=8$ TeV with the ATLAS detector, Phys. Rev. D 91 (2015) 012008 [arXiv: 1411.1559] [INSPIRE].

[37] CMS collaboration, Search for jet extinction in the inclusive jet- $p_{t}$ spectrum from proton-proton collisions at $\sqrt{s}=8$ TeV, Phys. Rev. D 90 (2014) 032005 [arXiv:1405.7653] [INSPIRE]. 
[38] M. Nagano and A.A. Watson, Observations and implications of the ultrahigh-energy cosmic rays, Rev. Mod. Phys. 72 (2000) 689 [InSPIRE].

[39] J.L. Feng and A.D. Shapere, Black hole production by cosmic rays, Phys. Rev. Lett. 88 (2002) 021303 [hep-ph/0109106] [INSPIRE].

[40] D. Kazanas and A. Nicolaidis, Cosmic rays and large extra dimensions, Gen. Rel. Grav. 35 (2003) 1117 [hep-ph/0109247] [INSPIRE].

[41] L. Anchordoqui and H. Goldberg, Experimental signature for black hole production in neutrino air showers, Phys. Rev. D 65 (2002) 047502 [hep-ph/0109242] [INSPIRE].

[42] Y. Uehara, Production and detection of black holes at neutrino array, Prog. Theor. Phys. 107 (2002) 621 [hep-ph/0110382] [INSPIRE].

[43] M. Kowalski, A. Ringwald and H. Tu, Black holes at neutrino telescopes, Phys. Lett. B 529 (2002) 1 [hep-ph/0201139] [INSPIRE].

[44] J. Alvarez-Muniz, J.L. Feng, F. Halzen, T. Han and D. Hooper, Detecting microscopic black holes with neutrino telescopes, Phys. Rev. D 65 (2002) 124015 [hep-ph/0202081] [INSPIRE].

[45] L.A. Anchordoqui, J.L. Feng, H. Goldberg and A.D. Shapere, Black holes from cosmic rays: probes of extra dimensions and new limits on TeV scale gravity, Phys. Rev. D 65 (2002) 124027 [hep-ph/0112247] [INSPIRE].

[46] ATLAS collaboration, Search for microscopic black holes and string balls in final states with leptons and jets with the ATLAS detector at $\sqrt{s}=8$ TeV, JHEP 08 (2014) 103 [arXiv: 1405.4254] [INSPIRE].

[47] ATLAS collaboration, Search for low-scale gravity signatures in multi-jet final states with the ATLAS detector at $\sqrt{s}=8 \mathrm{TeV}$, JHEP 07 (2015) 032 [arXiv: 1503.08988] [INSPIRE].

[48] ATLAS collaboration, Search for evidence for strong gravity in jet final states produced in pp collisions at $\sqrt{s}=13 \mathrm{TeV}$ using the ATLAS detector at the LHC, ATLAS-CONF-2015-043 (2015).

[49] K.S. Thorne, Nonspherical gravitational collapse - A short review, in Magic without magic: John Archibald Wheeler, J. Klauder ed., Freeman, San Francisco U.S.A. (1972).

[50] R.C. Myers and M.J. Perry, Black holes in higher dimensional space-times, Annals Phys. 172 (1986) 304 [INSPIRE].

[51] M. Cavaglia, Black hole and brane production in TeV gravity: a review, Int. J. Mod. Phys. A 18 (2003) 1843 [hep-ph/0210296] [INSPIRE].

[52] P. Kanti, Black holes in theories with large extra dimensions: a review, Int. J. Mod. Phys. A 19 (2004) 4899 [hep-ph/0402168] [INSPIRE].

[53] D.M. Gingrich and K. Martell, Study of highly-excited string states at the Large Hadron Collider, Phys. Rev. D 78 (2008) 115009 [arXiv:0808.2512] [INSPIRE].

[54] G. Landsberg, Black holes at future colliders and beyond, J. Phys. G 32 (2006) R337 [hep-ph/0205174].

[55] S. Dimopoulos and G. Landsberg, Black hole production at future colliders, in the proceedings of the APS/DPF/DPB Summer Study on the Future of Particle Physics (Snowmass 2011), June 30-July 21, Snowmass, Colorado, U.S.A. (2001), eConf (2011) C010630 P231.

[56] C.M. Harris, P. Richardson and B.R. Webber, CHARYBDIS: a black hole event generator, JHEP 08 (2003) 033. 
[57] J.A. Frost et al., Phenomenology of production and decay of spinning extra-dimensional black holes at hadron colliders, JHEP 10 (2009) 014.

[58] D.-C. Dai, G. Starkman, D. Stojkovic, C. Issever, E. Rizvi and J. Tseng, BlackMax: a black-hole event generator with rotation, recoil, split branes and brane tension, Phys. Rev. D 77 (2008) 076007 [arXiv: 0711.3012] [INSPIRE].

[59] D.-C. Dai, C. Issever, E. Rizvi, G. Starkman, D. Stojkovic and J. Tseng, Manual of BlackMax, a black-hole event generator with rotation, recoil, split branes and brane tension, arXiv:0902. 3577 [INSPIRE].

[60] D.M. Gingrich, Monte Carlo event generator for black hole production and decay in proton-proton collisions, Comput. Phys. Commun. 181 (2010) 1917 [arXiv:0911.5370] [INSPIRE].

[61] J. Tanaka, T. Yamamura, S. Asai and J. Kanzaki, Study of black holes with the ATLAS detector at the LHC, Eur. Phys. J. C 41 (2005) 19 [hep-ph/0411095] [INSPIRE].

[62] H. Yoshino and Y. Nambu, Black hole formation in the grazing collision of high-energy particles, Phys. Rev. D 67 (2003) 024009 [gr-qc/0209003] [INSPIRE].

[63] H. Yoshino and V.S. Rychkov, Improved analysis of black hole formation in high-energy particle collisions, Phys. Rev. D 71 (2005) 104028 [Erratum ibid. D 77 (2008) 089905] [hep-th/0503171] [INSPIRE].

[64] T. Sjostrand, S. Mrenna and P. Skands, PYTHIA 6.4 physics and manual, JHEP 05 (2006) 026.

[65] E. Boos et al., Generic user process interface for event generators, hep-ph/0109068 [INSPIRE].

[66] G. Landsberg, private communication.

[67] C.M. Harris, P. Richardson and B.R. Webber, CHARYBDIS: a black hole event generator, JHEP 08 (2003) 033.

[68] J.A. Frost et al., Phenomenology of production and decay of spinning extra-dimensional black holes at hadron colliders, JHEP 10 (2009) 014.

[69] D.-C. Dai, G. Starkman, D. Stojkovic, C. Issever, E. Rizvi and J. Tseng, BlackMax: a black-hole event generator with rotation, recoil, split branes and brane tension, Phys. Rev. D 77 (2008) 076007 [arXiv: 0711.3012] [INSPIRE].

[70] D.-C. Dai, C. Issever, E. Rizvi, G. Starkman, D. Stojkovic and J. Tseng, Manual of BlackMax, a black-hole event generator with rotation, recoil, split branes and brane tension, arXiv:0902.3577 [INSPIRE].

[71] M. Cavaglia, S. Das and R. Maartens, Will we observe black holes at the LHC?, Class. Quant. Grav. 20 (2003) L205.

[72] M. Cavaglia and S. Das, How classical are TeV scale black holes?, Class. Quant. Grav. 21 (2004) 4511 [hep-th/0404050] [INSPIRE].

[73] http://researchcomputing.ua.edu/.

[74] Alabama Supercomputer Authority, http://www.asc.edu/. 